\title{
ACTIVE-ONLY SINUSOIDAL OSCILLATOR CIRCUITS
}

\author{
MUHAMMAD TAHER ABUELMAATTI and MUHAMMAD \\ ALI AL-QAHTANI
}

King Fahd University of Petroleum and Minerals, Box 203, Dhahran 31261, Saudi Arabia

(Received 14 September 2001; In final form 6 November 2001)

Two new active-only sinusoidal oscillator circuits are proposed. The first circuit uses one current feedback operational amplifier (CFOA) and four operational transconductance amplifiers (OTAs). The second circuit uses only four OTAs. Without using any external passive elements, voltage (or current) control of the frequency and condition of oscillation, of the two circuits, can be achieved by adjusting the bias currents of the OTAs. The proposed circuits enjoy low sensitivities.

\section{INTRODUCTION}

At present, there is a growing interest in designing capacitor-less/ resistor-less active-filters using only active-elements, such as opertational amplifiers (OAs) and operational transconductance amplifiers (OTAs). This is attributed to their integratability, programmability and wide frequency range of operation (Abuelmaatti and Alzaher, 1997; Tsukutani etal., 1996).

Active-only resistor-less/capacitor-less sinusoidal oscillator circuits using two operational amplifiers have, also, been reported (Abuelmaatti and Almansoury, 1986; Bhat and Shah, 1989). The main disadvantage of these oscillators, is that there is no control on the condition of oscillation. Moreover, the frequency of oscillation can be controlled only by changing the dc supply of the whole circuit.

\footnotetext{
${ }^{*}$ Corresponding author.
} 
In this paper, new capacitor-less/resistor-less active-only sinusoidal oscillator circuits are presented. The first circuit uses four OTAs and one CFOA, and the second circuit uses only four OTAs. Both circuits enjoy independent current(voltage)-control of the frequency and the condition of oscillation.

\section{PROPOSED CIRCUIT}

Figure 1 shows the proposed circuits. OTAs and CFOAs are nonideal devices. Figure 2 shows the simplified models of the OTA (Urbas and Osiowski, 1982) and the CFOA (Svoboda, 1994). Using the models of Figure 2, the equivalent circuits of the oscillator circuits of Figure 1, can be represented by the circuits of Figure 3 .

Figure 3(a) shows the equivalent circuit of the oscillator circuit of Figure 1(a) with

$$
\begin{aligned}
& Y_{1}=g_{\mathrm{m} 1}+\frac{1}{R_{1}}+s C_{1} \\
& Y_{2}=-g_{\mathrm{m} 2}+\frac{1}{R_{2}}+s C_{2}
\end{aligned}
$$

and

$$
Y_{3}=g_{\mathrm{m} 3}+\frac{1}{R_{3}}+s C_{3}
$$

where

$$
\begin{aligned}
& C_{1}=C_{\mathrm{o} 1}+C_{\mathrm{o} 4} \\
& R_{1}=R_{\mathrm{i} 1} / / R_{\mathrm{o} 1} / / R_{\mathrm{o} 4} / / R_{y} \\
& C_{2}=R_{\mathrm{i} 2}+C_{\mathrm{o} 2} \\
& R_{2}=R_{\mathrm{i} 2} / / R_{\mathrm{o} 2} \\
& C_{3}=C_{t}+C_{\mathrm{i} 3}+C_{\mathrm{o} 3} \\
& R_{3}=R_{t} / / R_{\mathrm{i} 3} / / R_{\mathrm{o} 3}
\end{aligned}
$$

and

$$
g_{\mathrm{m} i}=\frac{I_{\mathrm{ABC} i}}{2 V_{T}}, \quad i=1-4
$$



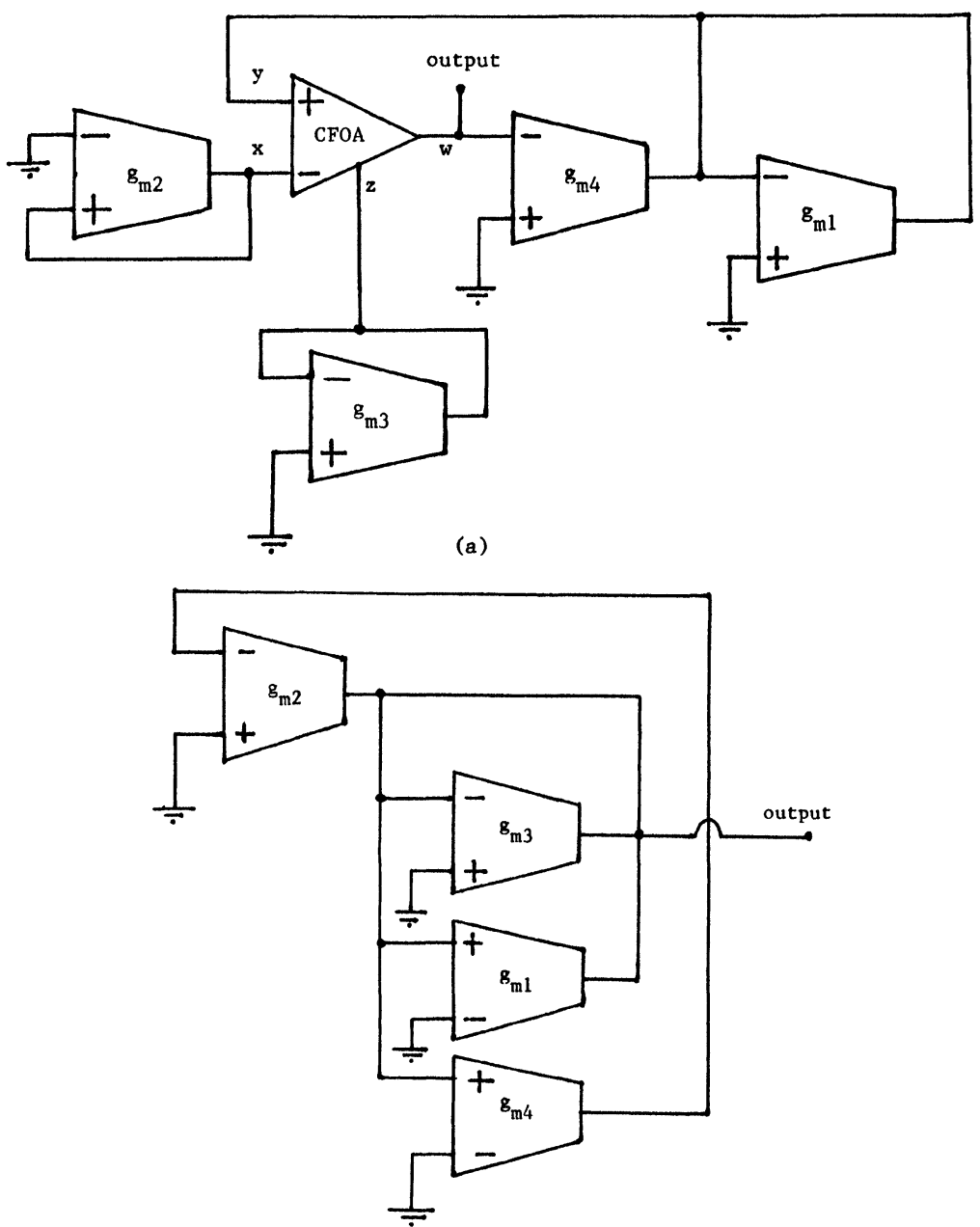

(b)

FIGURE 1 Proposed active-only oscillator circuits.

is the transconductance of the $i$ th OTA with $I_{\mathrm{ABC} i}=$ the auxiliary bias current of the $i$ th OTA and $V_{T}$ is the thermal voltage.

Using Figure 3(a) the characteristic equation of the oscillator circuit of Figure 1(a) can be expressed as

$$
\alpha \beta g_{\mathrm{m} 4} Y_{2}=Y_{1} Y_{3}\left(1+R_{x} Y_{2}\right)
$$



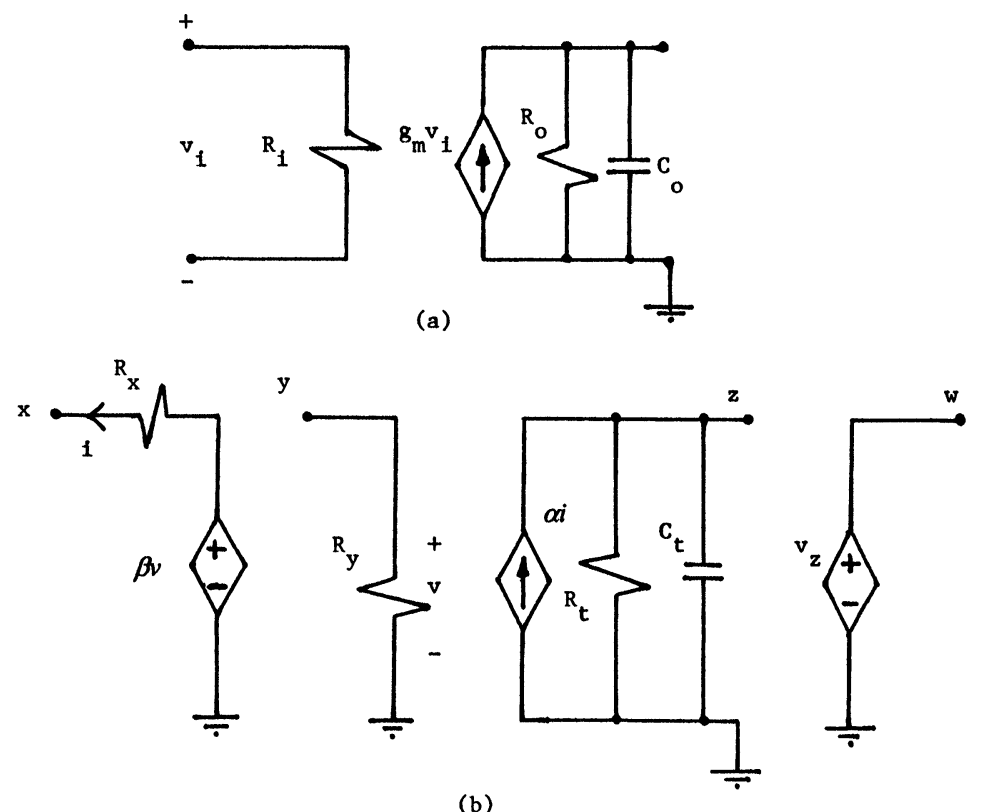

FIGURE 2 Simplified equivalent circuits for (a) the OTA; Urbas and Osiowski, 1982 (b) the CFOA; Svoboda, 1994.

where $\alpha$ and $\beta$ represent the current- and voltage-tracking errors of the CFOA. Assuming that $R_{x} Y_{2} \ll 1$, combining Eqs. (1)-(4), and applying the Barkhausen principle, the frequency of oscillation and the condition of oscillation of the oscillator circuit of Figure 1(a) can be expressed as

$$
\omega_{o}^{2}=\frac{1}{C_{1} C_{3}}\left(\left(g_{\mathrm{m} 1}+\frac{1}{R_{1}}\right)\left(g_{\mathrm{m} 3}+\frac{1}{R_{3}}\right)+\alpha \beta g_{\mathrm{m} 4}\left(g_{\mathrm{m} 2}-\frac{1}{R_{2}}\right)\right)
$$

and

$$
\alpha \beta g_{\mathrm{m} 4} C_{2}=\left(g_{\mathrm{ml}}+\frac{1}{R_{1}}\right) C_{3}+\left(g_{\mathrm{m} 3}+\frac{1}{R_{3}}\right) C_{1}
$$




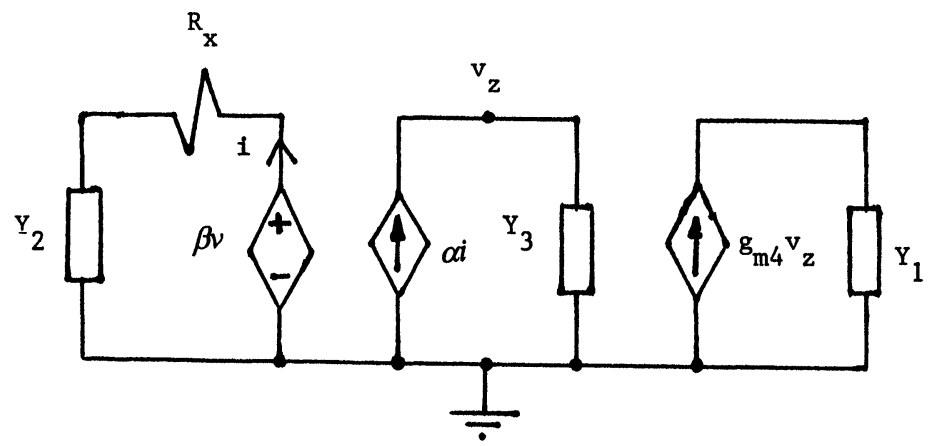

(a)

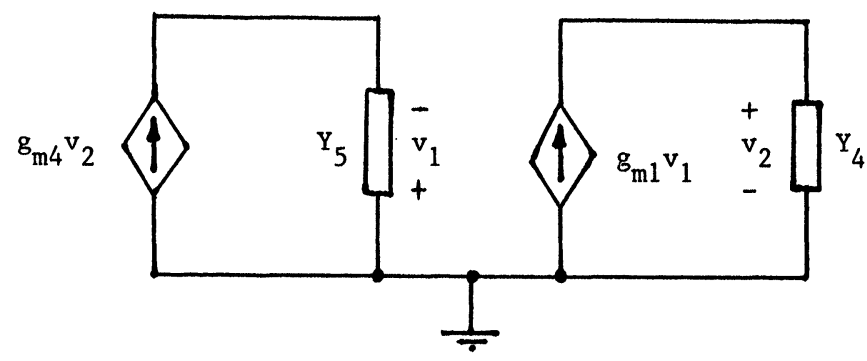

(b)

FIGURE 3 Equivalent circuits for the proposed active-only oscillator circuits of Figure 1.

With $1 / R_{1} \ll g_{\mathrm{m} 1}, 1 / R_{3} \ll g_{\mathrm{m} 3}$ and $1 / R_{2} \ll g_{\mathrm{m} 2}$, Eqs. (5) and (6) reduce to

$$
\omega_{o}^{2} \cong \frac{g_{\mathrm{m} 1} g_{\mathrm{m} 3}+\alpha \beta g_{\mathrm{m} 2} g_{\mathrm{m} 4}}{C_{1} C_{3}}
$$

and

$$
\alpha \beta g_{\mathrm{m} 4} C_{2} \cong g_{\mathrm{m} 1} C_{3}+g_{\mathrm{m} 3} C_{1}
$$

From Eqs. (5)-(8) it is easy to see that the frequency of oscillation can be controlled by adjusting $g_{\mathrm{m} 2}$ without disturbing the condition of oscillation, and the condition of oscillation can be controlled by adjusting $C_{2}$ without disturbing the frequency of oscillation. Thus, the circuit of Figure 1(a) enjoys independent control of the frequency and the condition of oscillation. 
Figure 3(b) shows the equivalent circuit of the oscillator circuit of Figure 1(b) with

$$
Y_{4}=g_{\mathrm{m} 2}-g_{\mathrm{m} 3}+\frac{1}{R_{1}}+s C_{1}
$$

and

$$
Y_{5}=\frac{1}{R_{2}}+s C_{2}
$$

where

$$
\begin{aligned}
& C_{1}=C_{\mathrm{o} 1}+C_{\mathrm{o} 2}+C_{\mathrm{o} 3} \\
& R_{1}=R_{\mathrm{i} 1} / / R_{\mathrm{i} 3} / / R_{\mathrm{i} 4} / / R_{\mathrm{o} 1} / / R_{\mathrm{o} 2} / / R_{\mathrm{o} 3} \\
& C_{2}=C_{\mathrm{o} 4}
\end{aligned}
$$

and

$$
R_{2}=R_{\mathrm{i} 2} / / R_{\mathrm{0} 4}
$$

Using Figure 3(b) the characteristic equation of the oscillator circuit of Figure 1(b) can be expressed as

$$
g_{\mathrm{m} 1} g_{\mathrm{m} 4}=-Y_{4} Y_{5}
$$

Combining Eqs. (9)-(11), and applying the Barkhausen principle, the frequency of oscillation and the condition of oscillation of the oscillator circuit of Figure 1(b) can be expressed as

$$
\omega_{o}^{2}=\frac{1}{C_{1} C_{2}}\left(g_{\mathrm{m} 1} g_{\mathrm{m} 4}+\frac{1}{R_{2}}\left(\frac{1}{R_{1}}+g_{\mathrm{m} 2}-g_{\mathrm{m} 3}\right)\right)
$$

and

$$
g_{\mathrm{m} 3} C_{2}=\left(g_{\mathrm{m} 2}+\frac{1}{R_{1}}\right) C_{2}+\frac{1}{R_{2}} C_{1}
$$


With $1 / R_{1} \ll g_{\mathrm{m} 2}-g_{\mathrm{m} 3}$, Eqs. (12) and (13) reduce to

$$
\omega_{o}^{2} \cong \frac{g_{\mathrm{m} 1} g_{\mathrm{m} 4}}{C_{1} C_{2}}
$$

and

$$
g_{\mathrm{m} 2} \cong g_{\mathrm{m} 3}
$$

From Eqs. (12)-(15) it is easy to see that the frequency of oscillation can be controlled by adjusting $g_{\mathrm{m} 1}$ and/or $g_{\mathrm{m} 4}$ without disturbing the condition of oscillation, and the condition of oscillation can be controlled by adjusting $g_{\mathrm{m} 2}$ and/or $g_{\mathrm{m} 3}$ without disturbing the frequency of oscillation. Thus, the circuit of Figure. 1(b) enjoys independent control of the frequency and the condition of oscillation.

From Eqs. (7) and (14) it is easy to show that the sensitivities of the parameter $\omega_{o}$ are

$$
\begin{aligned}
S_{C_{1}}^{\omega_{o}} & =S_{C_{3}}^{\omega_{o}}=-\frac{1}{2} \\
S_{g_{\mathrm{m} 1}}^{\omega_{o}} & =S_{g_{\mathrm{m} 3}}^{\omega_{o}}=\frac{1}{2} \frac{g_{\mathrm{m} 1} g_{\mathrm{m} 3}}{g_{\mathrm{m} 1} g_{\mathrm{m} 3}+\alpha \beta g_{\mathrm{m} 2} g_{\mathrm{m} 4}}
\end{aligned}
$$

and

$$
S_{\alpha}^{\omega_{o}}=S_{\beta}^{\omega_{o}}=S_{g_{\mathrm{m} 2}}^{\omega_{o}}=S_{g_{\mathrm{m} 4}}^{\omega_{o}}=\frac{1}{2} \frac{\alpha \beta g_{\mathrm{m} 2} g_{\mathrm{m} 4}}{g_{\mathrm{m} 1} g_{\mathrm{m} 3}+\alpha \beta g_{\mathrm{m} 2} g_{\mathrm{m} 4}}
$$

for the circuit of Figure 1(a), and

$$
S_{g_{\mathrm{m} 1}}^{\omega_{o}}=S_{g_{\mathrm{m} 4}}^{\omega_{o}}=-S_{C_{1}}^{\omega_{o}}=-S_{C_{2}}^{\omega_{o}}=\frac{1}{2}
$$

and

$$
S_{g_{\mathrm{m} 2}}^{\omega_{o}}=S_{g_{\mathrm{m} 3}}^{\omega_{o}}=0
$$

for the circuit of Figure 1(b).

Thus, all the $\omega_{o}$-sensitivities, of the circuits of Figure 1, are small. 


\section{Simulation Results}

The sinusoidal oscillator circuits of Figure 1 were simulated using the PSPICE circuit simulation program. The OTA macromodel of Figure 2(a) with $R_{i}=65 \mathrm{k} \Omega, R_{o}=63 \mathrm{M} \Omega, C_{o}=7.5 \mathrm{pF}$ and CFOA macromodel of Figure 2(b) with $R_{x}=50 \Omega, \quad R_{y}=10 \mathrm{M} \Omega$, $R_{t}=3 \mathrm{M} \Omega, C_{t}=4.5 \mathrm{pF}, \alpha=\beta=1$ were used in simulation. To avoid the possible latch-up and to limit the amplitude of oscillation, two anti-parallel biased-diodes were connected between the output terminal of the oscillator circuit and the ground. The results reported here were obtained with $\pm 2 \mathrm{~V}$ biasing voltage for the diodes.

The results obtained from the oscillator circuit of Figure 1(a) with $g_{\mathrm{ml}}=g_{\mathrm{m} 2}=g_{\mathrm{m} 3}=0.1 \mathrm{~mA} / \mathrm{V}, g_{m 4}=1.45 \mathrm{~mA} / \mathrm{V}$ are shown in Figure 4(a). Using Eq. (7) the calculated frequency of oscillation is $2.77 \mathrm{MHz}$ and the simulated frequency obtained from Figure $4(\mathrm{a})$ is $2.742 \mathrm{MHz}$. It appears that the simulated and calculated results are in good agreement. This justifies the approximations made in obtaining Eq. (7).

The results obtained from the oscillator circuit of Figure 1(b) with $g_{\mathrm{m} 1}=g_{\mathrm{m} 2}=6 \mathrm{~mA} / \mathrm{V}, g_{\mathrm{m} 3}=g_{\mathrm{m} 4}=7 \mathrm{~mA} / \mathrm{V}$ are shown in Figure 4(b). Using Eq. (14) the calculated frequency of oscillation is $79.4 \mathrm{MHz}$ and the simulated frequency obtained from Figure $4(b)$ is $77.6 \mathrm{MHz}$. It appears that the simulated and calculated results are in good agreement. This justifies the approximations made in obtaining Eq. (14).

\section{CONCLUSION}

Two new active-only sinusoidal oscillator has been presented. The first circuit, of Figure 1(a), uses one current feedback operational amplifier and four operational transconductance amplifiers. The second circuit, of Figure 1(b), uses only four operational transconductance amplifiers. No external passive elements are used. The second oscillator circuit enjoys the following advantages:

a. independent current control of the frequency and the condition of oscillation.

b. low active and passive sensitivities of the frequency of oscillation. 

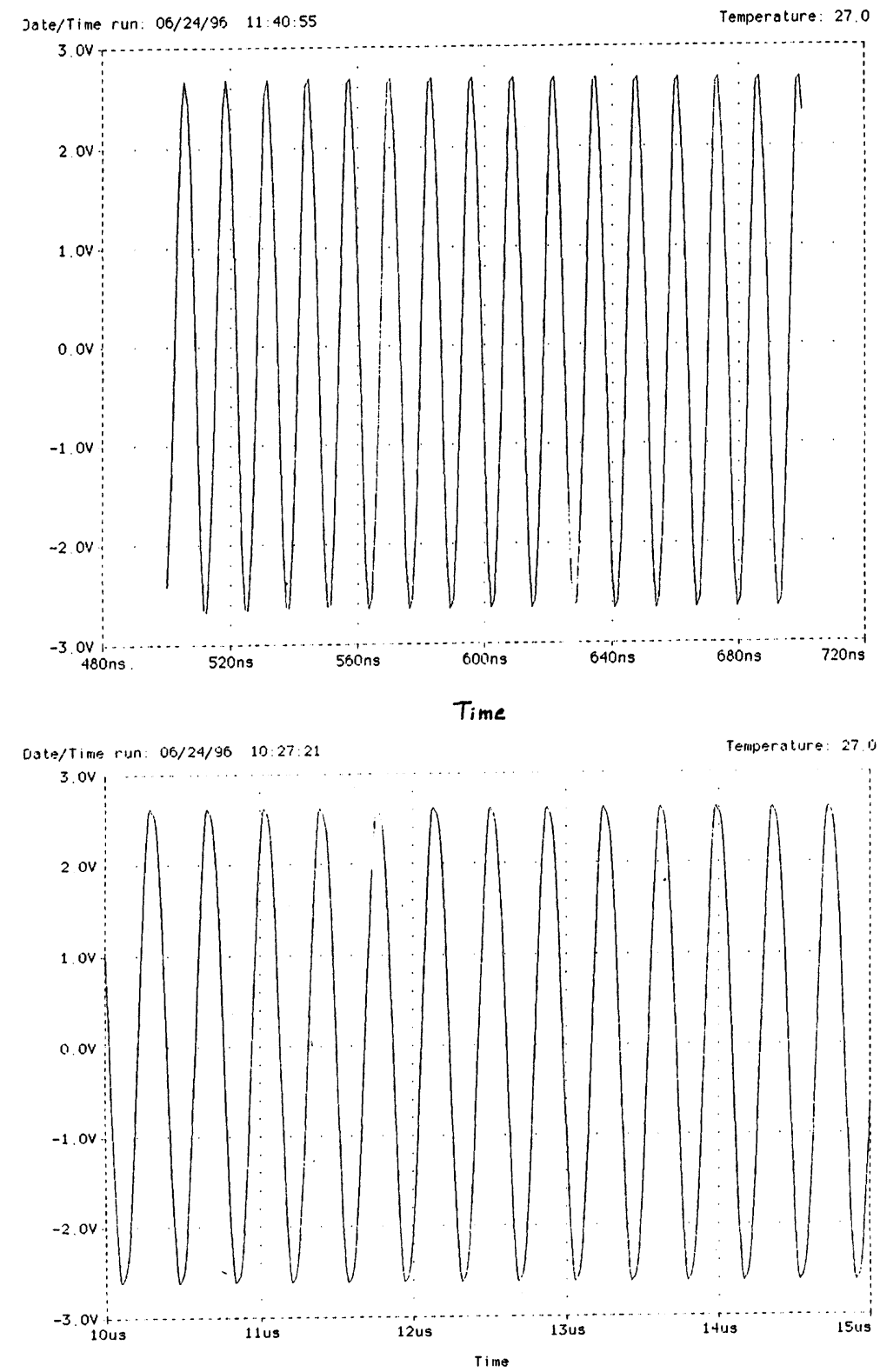

FIGURE 4 Simulation results obtained from (a) the oscillator circuit of Figure 1(a) with $g_{\mathrm{m} 1}=g_{\mathrm{m} 2}=g_{\mathrm{m} 3}=0.1 \mathrm{~mA} / \mathrm{V}, g_{\mathrm{m} 4}=1.45 \mathrm{~mA} / \mathrm{V}$ and (b) the oscillator circuit of Figure 1(b) with $g_{\mathrm{m} 1}=g_{\mathrm{m} 2}=6 \mathrm{~mA} / \mathrm{V}, g_{\mathrm{m} 3}=g_{\mathrm{m} 4}=7 \mathrm{~mA} / \mathrm{V}$. 
However, the output impedance of this circuit is high. This can be solved using the on-chip unity-gain buffer available with the OTA.

The first circuit uses an additional CFOA and enjoys a low output impedance terminal. While its frequency of oscillation can be adjusted using the auxiliary bias current of an OTA without disturbing the condition of oscillation, the condition of oscillation cannot be adjusted using the auxiliary bias current of an OTA.

\section{References}

Abuelmaatti, M. T. and Almansoury, W. A. (1986). New active-R sinusoidal oscillator. International Journal of Electronics, 60, 771-775.

Abuelmaatti, M. T. and Alzaher, H. A. (1997). Universal three input and one output current-mode filter without external passive elements. Electronics Letters, 33, 281 283.

Bhat, C. K. and Shah, N. A. (1989). A novel voltage controlled oscillator. Electronic Engineering, 61(10), 25-26.

Svoboda, J. A. (1994). Comparison of RC op.amp. and RC current conveyor filters. International Journal of Electronics, 76, 615-626.

Tsukutani, T., Ishida, M., Tsuiki, S. and Fukui, Y. (1996). Current-mode biquad without external passive elements. Electronics Letters, 32, 197-198.

Urbas, A. and Osiowski, J. (1982). High frequency realization of C-OTA second order active filters. IEEE International Symposium on Circuits and Systems, pp. 11061109. 

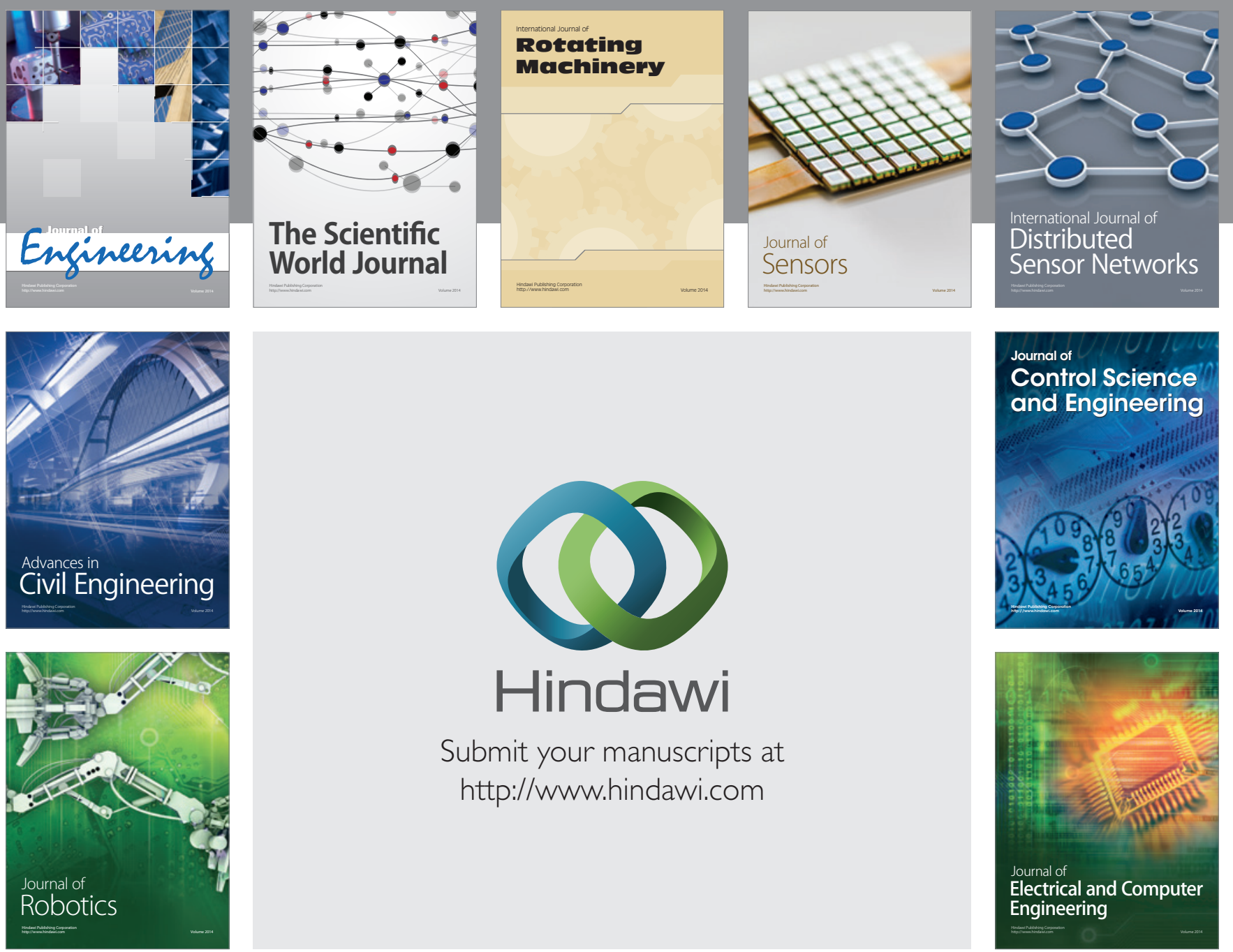

Submit your manuscripts at

http://www.hindawi.com
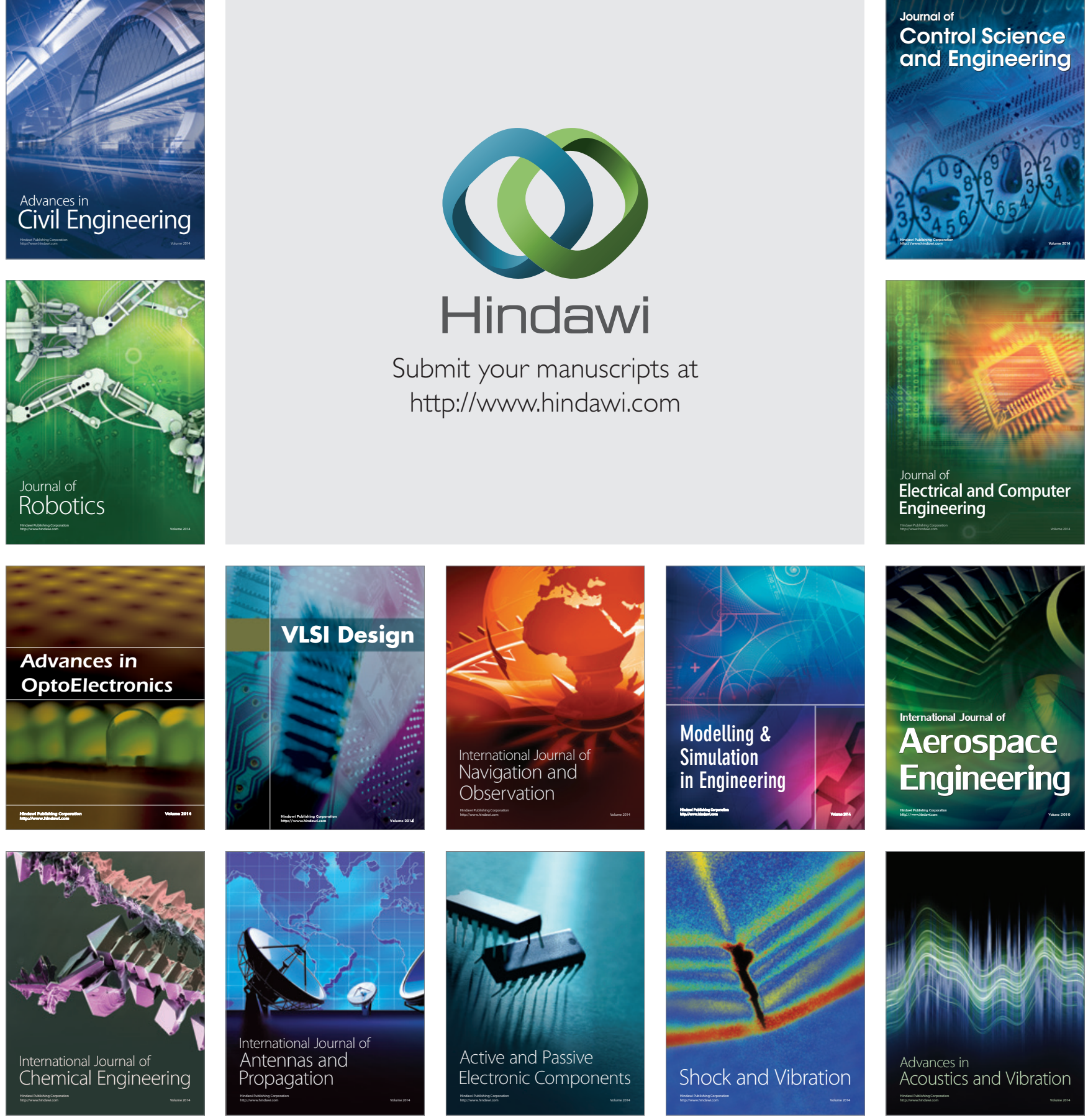